\title{
On the Pfaffian Invariant of a Skew-Symmetric Matrix ${ }^{1}$
}

\author{
By H. SCHWERDTFEGER
}

(Received 25th November, 1946. Read 6th December, 1946.)

1. Let $P$ be an $n$-rowed skew-symmetric matrix of rank $2 r$ with elements out of an infinite field $F$. Denoting by $x, y$ columns of $n$ variables (indeterminates over $F)^{\prime} x_{\nu}, y_{v}(v=1, \ldots, n)$, and by $x^{\prime}, y^{\prime}$ the corresponding row matrices, we consider the skew-symmetric bilinear form $y^{\prime} P x$. It is well known that for every $P$ a regular homo. geneous substitution $x=T \bar{x}, y=T \bar{y}$ over $F$ can be found so that

$$
\begin{aligned}
y^{\prime} P x & =\bar{x}_{1} \bar{y}_{2}-\bar{x}_{2} \bar{y}_{1}+\ldots \bar{x}_{2 r-1} \bar{y}_{2 r}-\bar{x}_{2 r} \bar{y}_{2 r-1} \\
& =\bar{y}^{\prime} I_{r} \bar{x} .
\end{aligned}
$$

In the new coordinates the form has the matrix

$$
I_{r}=T^{\prime} P T,
$$

which may be considered as "congruence normal form" of any $P$ of rank $2 r$. The normal form is independent of the choice of the ground field $F$; it is the diagonal array of $r$ two-rowed matrices $\left(\begin{array}{lr}0 & -1 \\ 1 & 0\end{array}\right)$, all other elements of the matrix $I_{r}$ being zeros.

Further, let $u, v$ be two column matrices with $n$ coordinates each; it is readily seen that

$$
[u, v]=v u^{\prime}-u v^{\prime}
$$

is a skew-symmetric matrix, of rank 2 if and only if the two columns $u, v$ are linearly independent, otherwise zero. The symbol $[u, v]$ obeys the congruence transformation law

$$
T^{\prime}[u, v] T=\left[T^{\prime} u, T^{\prime} v\right]
$$

Its congruence normal form is the matrix $\left[e^{(1)}, e^{(2)}\right]$ where $e^{(v)}$ is the unit column having $a 1$ in the $\nu$-th place and zeros in all other places. Since $\left[e^{(1)}, e^{(2)}\right]$ is also the congruence normal form of any given skewsymmetric matrix of rank 2, we see that by suitable choice of $u, v$ any such matrix can be represented in the form $[u, v]$.

Making use of this notation we have

$$
I_{r}=\left[e^{(1)}, e^{(2)}\right]+\ldots+\left[e^{(2 r-1)}, e^{(2 r)}\right] .
$$

1 Presented at the meeting of the Australian and New Zealand Association for the Advancement of Science, Adelaide, August, 1946. 
On the Pfaffian Invariant of a Skew-Symmetric Matrix 107

By applying to both sides of this equation the congruence transformation with the matrix $T^{-1}$ we obtain the theorem that every skew-symmetric matrix of rank $2 r$ is the sum of $r$ skew-symmetric matrices of rank 2 .

2. In particular let us suppose now that $P$ is regular, $|P| \neq 0$. Then $n$ is even: $n=2 m, m=r$, and according to the preceding statement there are $2 m$ columns $u_{(1)}, \ldots, u_{(2 m)}$ such that

$$
P=\left[u_{(1)}, u_{(2)}\right]+\ldots+\left[u_{(2 m-1)}, u_{(2 m)}\right] .
$$

A matrix $T$ can be found which, by congruence transformation, turns $P$ into its normal form $T^{\prime} P T=I_{m}=I$. so that

We introduce the matrix $U$ which has $u_{(1)}^{\prime}, \ldots, u_{(2 m)}^{\prime}$ as its rows

$$
U^{\prime}=\left(u_{(1)} u_{(2)} \ldots u_{(2 m-1)} u_{(2 m)}\right) .
$$

Then

$$
U^{\prime} I U=\left[U^{\prime} e^{(1)}, U^{\prime} e^{(2)}\right]+\ldots+\left[U^{\prime} e^{(2 m-1)}, U^{\prime} e^{(2 m)}\right]=P,
$$

because $U^{\prime} e^{(v)}=u_{(v)}$. Hence $T=U^{-1}$.

At the same time we notice the determinant relation

$$
|P|=|U|^{2} \text {. }
$$

Thus $|U|^{2}$ is uniquely defined by the matrix $P$. It is, however, a fact that also the determinant $|U|$ (including its sign). is uniquely defined by the matrix $P$. The determinant $|U|$ is called the Pfaffian of the matrix $P$.

3. In order to prove the uniqueness of the Pfaffian of $P$ we introduce the group of all matrices $S$ for which

$$
S^{\prime} P S=P
$$

this group is called the symplectic group of the matrix $P$. We shall need here only the fact that every symplectic matrix $S$ has its determinant $|S|=1$.

It is sufficient to prove this for a certain skew-symmetric matrix $P$ of rank $2 m$. We choose for $P$ the matrix

$$
J=\left[e^{(1)}, e^{(m+1)}\right]+\ldots+\left[e^{(m)}, e^{(2 m)}\right]=\left(\begin{array}{cc}
0 & -E \\
E & 0
\end{array}\right)
$$

where $E$ represents the $m$-rowed unit matrix. Since then $S^{\prime}$ as well as $S$ is a symplectic matrix we have at once the relation

$$
S J S^{\prime}=J
$$


which, if we set

$$
S=\left(\begin{array}{ll}
A & B \\
C & D
\end{array}\right)
$$

where $A, B, C, D$ are $m$-rowed square matrices, is easily resolved into the matrix equations

$$
\begin{gathered}
B A^{\prime}=A B^{\prime}, \quad D C^{\prime}=C D^{\prime}, \\
D A^{\prime}-C B^{\prime}=A D^{\prime}-B C^{\prime}=E . \\
S^{-1}=\left(\begin{array}{r}
D^{\prime}-B^{\prime} \\
-C^{\prime}
\end{array}\right) .
\end{gathered}
$$

Hence

Following C. L. Siegel ${ }^{1}$ we introduce now a pair of matrices $X, Y$ subject only to the condition $X Y^{\prime}=Y X^{\prime}$; then

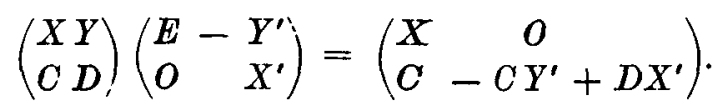

By intercalating between the two matrix factors on the left-hand side of this equation the matrix product $S^{-1} S=E$ we obtain the identity in the form

$$
\left(\begin{array}{cc}
X D^{\prime}-Y C^{\prime} & -X B^{\prime}+Y A^{\prime} \\
O & E
\end{array}\right) S\left(\begin{array}{cc}
E-Y^{\prime} \\
O & X^{\prime}
\end{array}\right)=\left(\begin{array}{cc}
X & O \\
C & -C Y^{\prime}+D X^{\prime}
\end{array}\right)
$$

Now we take the determinant of both sides:

$$
\left|X D^{\prime}-Y C^{\prime}\right||S||X|=|X|\left|-C Y^{\prime}+D X^{\prime}\right|
$$

Since $X=E$ is admissible, the determinant $|X|$ is not identically zero; and since

$$
\left|X D^{\prime}-Y C^{\prime}\right|=\left|-C Y^{\prime}+D X^{\prime}\right|
$$

is equal to 1 for $X=A, Y=B$, this determinant, also, cannot vanish identically; therefore $|S|=1$.

4. Now let $U$ be a fixed matrix such that $U^{\prime} I U=P$. If $S$ is a symplectic matrix for $P$, the matrix $U S=V$ has the same property as $U$, viz. $V^{\prime} I V=P$. Conversely every $V$ with this property can be written in the form $U S$ with a suitable symplectic $S$. In fact

$$
V^{\prime} I V=V^{\prime} U^{\prime-1} P U^{-1} V=\dot{P} \text {. }
$$

Thus $U^{-1} V=S$ is symplectic for $P$, and $V=U S$.

Hence we see that all $V$ have the determinant

$$
|V|=|U| \text {, }
$$

which proves the uniqueness of the Pfaffian of $P$.

1 Math. Annalen, 116 (1939), 620. 


\section{On the Pfaffian Invariant of A Skew-Symmetric Matrix 109}

Further we show that $|U|$ is an invariant of weight one. Since all regular $2 m$-rowed skew-symmetric matrices are congruent, there is, for any such matris $Q$, a regular matrix $R$ such that

$$
R^{\prime} P R=Q .
$$

Therefore

$$
Q=R^{\prime} U^{\prime} I U R \text {, }
$$

whence we see that

$$
|U R|=|U||R|
$$

is the Pfaffian of $R^{\prime} P R$.

Usually this invariance of the Pfaffian is employed in order to prove the theorem on the symplectic determinant. The inverse way proposed here seems to be worth noticing since Siegel's formal proof of the said theorem is very simple. ${ }^{1}$

5. With regard to applications in mechanics and optics the matrix $J=J_{2 m}$ is used by preference as the invariant matrix for the representation of the symplectic group; it is therefore convenient to refer to it as the normal form of a skew-symmetric matrix. We shall determine its Pfaffian.

The congruence transformation $T$ turning $J_{2 m}$ into $I$ is the permutation matrix which belongs to the permutation

$$
\pi_{m}=\left(\begin{array}{ccccccc}
1 & 2 & 3 & 4 & \ldots & 2 m-1 & 2 m \\
1 & m+1 & 2 & m+2 & m & 2 m
\end{array}\right) .
$$

The determinant of this matrix is +1 if $\pi_{m}$ is even, -1 if $\pi_{m}$ is odd. In the cycle notation we have

$$
\begin{aligned}
& \pi_{2}=\left(\begin{array}{ll}
2 & 3
\end{array}\right), \quad \pi_{3}=\left(\begin{array}{llll}
2 & 4 & 5 & 3
\end{array}\right), \quad \pi_{4}=\left(\begin{array}{llll}
2 & 5 & 3
\end{array}\right)\left(\begin{array}{llll}
4 & 6 & 7
\end{array}\right)
\end{aligned}
$$

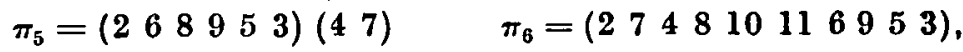

$$
\begin{aligned}
& \pi_{7}=\left(\begin{array}{lllllllll}
2 & 8 & 116 & 10 & 12 & 13 & 7495 & 4
\end{array}\right) \text {, etc. }
\end{aligned}
$$

Thus $\pi_{2}, \pi_{3}$ are odd, $\pi_{4}, \pi_{5}$ even, $\pi_{6}, \pi_{7}$ odd. To prove this law in general we notice that

$$
\pi_{m+1}=(m+1 \quad m+2 \ldots 2 m+1) \pi_{m} .
$$

Here the cycle factor on the right moves $m+1$ symbols; it is therefore an even permutation if $m$ is odd and conversely. Hence, for an even

1 Another formal proof of the theorem was attempted by the author in an earlier note on the subject (Proc. Roy. Soc., New South Wales, 76 (1942), 177-181). However, a lemma upon which the proof was based turned out to be false. 
$m$, there follows $\pi_{m}$ a $\pi_{m+1}$ of the same character, and then a $\pi_{m+2} \mathrm{c}$ the opposite character. Since $\pi_{4}$ is even

$$
\pi_{m} \text { is }\left\{\begin{array}{cc}
\text { even if } m=4 \mu & \text { or } 4 \mu+1 \\
\text { odd if } m=4 \mu+2 & \text { or } 4 \mu+3 .
\end{array}\right.
$$

Since the Pfaffian of $J_{2 m}$ has the sign of $\pi_{m}$ we have

$$
\operatorname{Pf}\left(J_{2 m}\right)=(-1)^{\left[\frac{m}{2}\right]} \text {. }
$$

Instead of the Pfaffian $\operatorname{Pf}(P)$ of a $2 m$-rowed skew-symmetri matrix $P$ one can therefore also consider the determinant of th matrix $\bar{U}$ for which $\bar{U}^{\prime} J \bar{U}=P$. Then the Pfaffian of $P$ is

$$
\operatorname{Pf}(P)=|U|=(-1)^{\left[\frac{m}{2}\right]}|\bar{U}|
$$

6. With two $2 m$-rowed skew-symmetric matrices $P, Q$ we conside the linear combination $\lambda P-Q$ where $\lambda$ is a scalar parameter. If is regular, the Pfaffian

$$
\kappa(\lambda)=\operatorname{Pf}(\lambda P-Q)
$$

is a polynomial of degree $m$ in $\lambda$ since the Pfaffian is rational in th matrix elements and its square equal to the determinant

$$
|\lambda P-Q|=|P|\left|\lambda E-P^{-1} Q\right|=|P| \lambda^{2 m}+\ldots
$$

Evidently $\quad \kappa(\lambda)=\operatorname{Pf}(P) \operatorname{Pf}(\lambda I-\widetilde{Q}) \quad\left(U^{\prime} \widetilde{Q} U=Q\right)$.

Thus if $\quad \kappa(\lambda)=k_{0} \lambda^{m}+k_{1} \lambda^{m-1}+\ldots+k_{m}$,

the coefficients $k_{0}, k_{1}, \ldots, k_{m}$ form a set of simultaneous rational cor gruence invariants of $P$ and $Q$, which is, at the same time, a set c similarity invariants of the matrix $P^{-i} Q$.

In the theory of skew-symmetric matrices the polynomial $\kappa(i$ takes over the role of the characteristic polynomial $|\lambda E-A|=f($ i in the case of a general matrix $A$. Let $h(\lambda)$ be the highest invarian factor of $\lambda P-Q$; then $h(\lambda)^{2}$ is a divisor of $|\lambda P-Q|$, and therefor $h(\lambda)$ a divisor of $\kappa(\lambda)$. Thus we have ${ }^{1}$

$$
\kappa\left(P-{ }^{1} Q\right)=0,
$$

in analogy with the general Cayley identity $f(A)=0$.

1 Cf. H. Schwerdtfeger, American Math. Monthly, 51 (1944), 141.

Univerisity of Melbodrne, Australia. 